Nota científica

(Short communication)

\title{
NUEVOS REGISTROS DE LA COMADREJA COLA LARGA (MUSTELA FRENATA) EN LA ZONA SEMIDESÉRTICA DE DURANGO, MÉXICO \author{
DESERT ZONE OF DURANGO, MEXICO
} \\ NEW RECORDS OF THE LONG-TAILED WEASEL (MUSTELA FRENATA) IN THE SEMI-
}

\section{LUIS GERARDO YÁÑEZ CHÁVEZ, MAYELA RODRÍGUEZ GONZÁLEZ*, GONZALO HERNÁNDEZ IBARRA, MALI NAY BUENDÍA TAMARIZ}
Unidad Regional Universitaria de Zonas Áridas de la Universidad Autónoma Chapingo. Km 40 carretera Gómez Palacio- Chihuahua. Bermejillo, Durango, 35230, México. <yachgl@ chapingo.uruza.edu.mx>; <maye.rodriguez@chapingo.uruza.edu.mx>; <chalonza2011@gmail.com>; <mali.buendia@ @otmail.com>
*Autor de correspondencia: <maye.rodriguez@chapingo.uruza.edu.mx>

Recibido: 09/05/2018; aceptado: 02/07/2019; publicado en línea: 11/07/2019

Editor responsable: Alberto González Romero

Yáñez-Chávez, L. G., Rodríguez-González, M., Hernández-Ibarra, G., Buendía-Tamariz, M. N. (2019) Nuevos registros de la comadreja cola larga (Mustela frenata) en la zona semidesértica de Durango, México. Acta Zoológica Mexicana (nueva serie), 35, 1-5. https://doi.org/10.21829/azm.2019.3501206

RESUMEN. Se reportan tres registros nuevos de la comadreja (Mustela frenata) para la zona semidesértica en el noreste del estado de Durango, mediante capturas fotográficas de individuos y georreferenciación, esto permite actualizar las observaciones de esta especie en la región.

Yáñez-Chávez, L. G., Rodríguez-González, M., Hernández-Ibarra, G., Buendía-Tamariz, M. N. (2019) New records of the long-tailed weasel (Mustela frenata) in the semi-desert zone of Durango, Mexico. Acta Zoológica Mexicana (nueva serie), 35, 1-5. https://doi.org/10.21829/azm.2019.3501206

ABSTRACT. We report three new records of the long-tailed weasel (Mustela frenata) for the semi-desert zone in the Northeast of the State of Durango, by means of photographic captures of the individuals and georeferencing, this allows to update the observations of this species in the region.

La comadreja cola larga (Mustela frenata Lichtenstein, 1831) es un carnívoro de la familia Mustelidae; se le encuentra desde el suroeste de Canadá hasta Argentina, en México se les halla en toda la República Mexicana, con excepción de la parte oeste de Sonora y la península de Baja California (Sheffield \& Thomas, 1997; Feldhamer et al., 2007). Por su tolerancia a condiciones ecológicas diversas y hábitos cosmopolitas, la comadreja cola larga aprovecha ambientes con distinto grado de alteración, desde vegetación natural, cultivos hasta ambientes urbanos, donde construye sus madrigueras subterráneas, también utiliza oquedades o madrigueras de otros animales (Gehring \& Swihart, 2004; Aranda, 2012). Mide entre 30 y $55 \mathrm{~cm}$ de longitud (Sheffield \& Thomas, 1997); su ámbito hogareño abarca de 12 a 16 hectáreas, consumen presas 
con peso entre 10 y $150 \mathrm{~g}$, incluyendo ratones, tuzas, ardillas, conejos y liebres, en ocasiones algunas lagartijas e invertebrados (Vaughan, 1985), especies abundantes en la zona semiárida de Durango. La comadreja cola larga no se encuentra enlistada en categorías de riesgo para México (SEMARNAT, 2010). Igualmente, la Unión Internacional para la Conservación de la Naturaleza (IUCN por sus siglas en inglés) la clasifica como especie de preocupación menor para su conservación (Helgen \& Reid, 2016).

Los mapas de distribución potencial de la especie muestran que es abundante en todo México, con áreas excluyentes de la península de Baja California y algunas zonas de Chihuahua, Sonora y Coahuila (Ceballos et al., 2006). Para el estado de Durango, Servín-Martínez et al. (1998), la incluye en el inventario de mamíferos, no obstante, sin indicar sitios específicos de las observaciones. Otros autores, la catalogan únicamente en la región ecológica de los valles de Durango, la cual corresponde a una amplia franja de tierras con clima cálido y seco, con dominancia de pastizales y mesetas (García-Mendoza \& LópezGonzález, 2013).

En Durango el consenso de distribución corre desde los valores más bajos hasta valores elevados, específicamente, para la zona semiárida del estado. Esta región natural se ubica al noreste de la entidad, está representada por llanuras y serranías del Bolsón de Mapimí. El clima prevalente es muy seco semicálido (BWh). La vegetación se compone de matorrales xerófilos, comunidades halófilas y gipsófilas. La topografía y los suelos son aptos para la agricultura, pero está se ve limitada por la falta de agua. La región alberga, sin embargo, importantes zonas agrícolas, gracias a los ríos Nazas y Aguanaval, procedentes de la Sierra Madre Occidental en Durango y Zacatecas (González et al., 2006). Hasta hoy, solo existe un registro documentado de la comadreja en esta región natural, la observación data de 1959 (Baker \& Greer, 1962); estos autores señalaron que aldeanos que vivían entre Lerdo y Tlahualilo, al noreste de Durango, indicaron que la comadreja era común observarla a lo largo de canales de riego adyacentes a los campos de cultivo; este registro fue georreferenciado por Servín y Ramos (2017). Martínez-Hernández y Rodríguez-Luévano (2017) también la señalan como especie potencial en Viesca, Coahuila, área cercana a Mapimí con condiciones climáticas y de vegetación similares. Asimismo, la CONANP (2006) reporta a este mamífero como especie potencial en la Reserva de la Biósfera Mapimí, situada en el centro del desierto Chihuahuense entre los estados de Durango, Coahuila y Chihuahua, aunque Elizalde-Arellano et al., (2014) no la registraron en esta área natural protegida.

El presente trabajo documenta y describe tres registros de comadreja cola larga para la zona semiárida del noreste del estado de Durango en las localidades de Bermejillo, La Victoria y URUZAChapingo (Fig. 1), obtenidos en el 2017 como parte de un estudio de monitoreo sobre fauna silvestre del área de influencia de la Universidad Autónoma Chapingo.

El área presenta un clima muy árido, semicálido con lluvias en verano, con temperaturas máximas y mínimas extremas en verano e invierno y una precipitación anual promedio de $240 \mathrm{~mm}$ (García, 2004). El relieve del área tiene una morfología de planicies con una altitud que varía entre los 1,100-1,300 m y con poca influencia de lomeríos, con una vegetación predominante de matorral desértico micrófilo y vegetación secundaria (INEGI, 2015), además se encuentran diferentes áreas perturbadas por el hombre que ahora son dedicadas para la agricultura de riego con presencia de extensos canales de riego revestidos de concreto y otros de tierra (acequias de terrado).

Durante las estaciones de verano y otoño del 2017 se realizaron 75 muestreos sistemáticos en un ecosistema de vegetación de matorral desértico micrófilo al sur de los municipios de Mapimí y Tlahualilo, Durango. Se utilizó un sistema de muestreo de observación directa por el método de transecto lineal, cada uno fue de $20 \mathrm{~km}$ de longitud por $50 \mathrm{~m}$ de ancho comprendiendo un área de 7,500 ha, los recorridos fueron entre las 07:00 y 21:00 horas. Cada individuo fue registrado con fotografías obtenidas con una cámara digital manual Coolpix B500, además las observaciones fueron georreferenciadas con un GPS Garmin Etrex Legend. Para los tres registros de comadrejas fue posible estimar el tamaño longitudinal, debido al rastro in 
situ de huellas, además de su etapa de desarrollo determinada por criterios morfométricos, de acuerdo con la metodología de Elsasser y Parker (2008), con ejemplares colectados.

El primer registro de comadreja fue obtenido el 4 de agosto del 2017 a las 13:00 horas (Fig. 2a), en la localidad URUZA-Chapingo (N 25 53' 28.78", W 103'36' 12.87", a una altitud de 1,115 m) cerca de un canal de riego revestido de concreto, la longitud aproximada del individuo fue de $50 \mathrm{~cm}$, con base en la medida de sus huellas trasera y delantera. El segundo registro se hizo el 28 de agosto del 2017 a las 19:23 horas a un costado de la carretera Bermejillo-Chapingo, el ejemplar estaba parado a la orilla de un terreno agrícola con alta densidad de zacate buffel (Pennisetum ciliare L.), (N 25 53' 20.1", W 103 36' 21.37", a una altitud de 1,120 m) el espécimen tenía una longitud cercana a los $44 \mathrm{~cm}$ (Fig. 2b). Ambos registros corresponden a individuos adultos, las fotografías no permiten conocer su sexo; la distancia entre estas observaciones fue de $362 \mathrm{~m}$. El tercer registro se logró documentar el 18 de septiembre del 2017 a las 20:06 horas cerca de la comunidad La Victoria, el animal se encontraba en un área con mezquitales (Prosopis spp.) y gobernadoras (Larrea tridentata) de porte bajo (N 25 53' 16.55", W $103^{\circ} 35^{\prime} 47.65^{\prime \prime}$, a una altitud de $1,113 \mathrm{~m}$ ). Este registro corresponde a un individuo juvenil con una longitud próxima a los $35 \mathrm{~cm}$, aunque no fue posible conocer su sexo (Fig. 2c); las distancias al primer y segundo registro fueron de 802 y $948 \mathrm{~m}$, respectivamente.

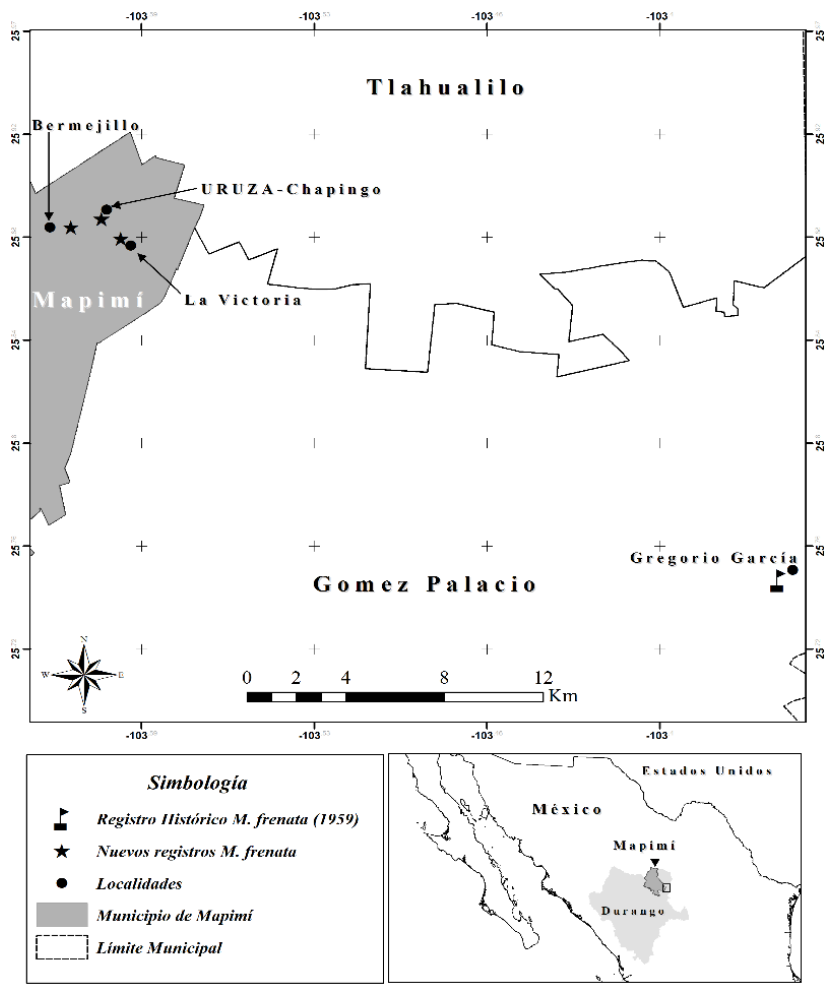

Figura 1. Localización del área de estudio y registros de la comadreja de cola larga (Mustela frenata) en la zona semiárida al noreste de Durango, México.

Los tres registros obtenidos en la zona semiárida del noreste de Durango, confirman la presencia y amplían el área de distribución de esta especie en la región a una distancia de $30 \mathrm{~km}$ respecto al histórico, ya que a pesar de que se consultaron diferentes bases de datos, se encontró solo un registro cercano a los 60 años de antigüedad. A pesar de que la comadreja cola larga está clasificada como especie de preocupación menor para la IUCN (Helgen \& Reid, 2016), el presente trabajo expone la relevancia de desarrollar estudios específicos sobre fauna silvestre en regiones poco exploradas, esto permitirá tener mayor certeza sobre la 
distribución real y potencial de las especies, sobre todo en escenarios de defaunación contemporánea (Dirzo et al., 2014). Adicionalmente, $M$. frenata puede ser sensible a la fragmentación del hábitat producida por la agricultura (Gehring \& Swihart, 2004). La importancia de las comadrejas radica en su eficiencia como depredadores de pequeños mamíferos y otros vertebrados, constituyendo un importante factor para la regulación de las poblaciones de los roedores que pueden tornarse en plagas, sin embargo, sus poblaciones generalmente fluctúan y pueden desaparecer localmente en respuesta al cambio en la cantidad de sus presas, la destrucción de su hábitat y uso de pesticidas para el control de plagas agrícolas.

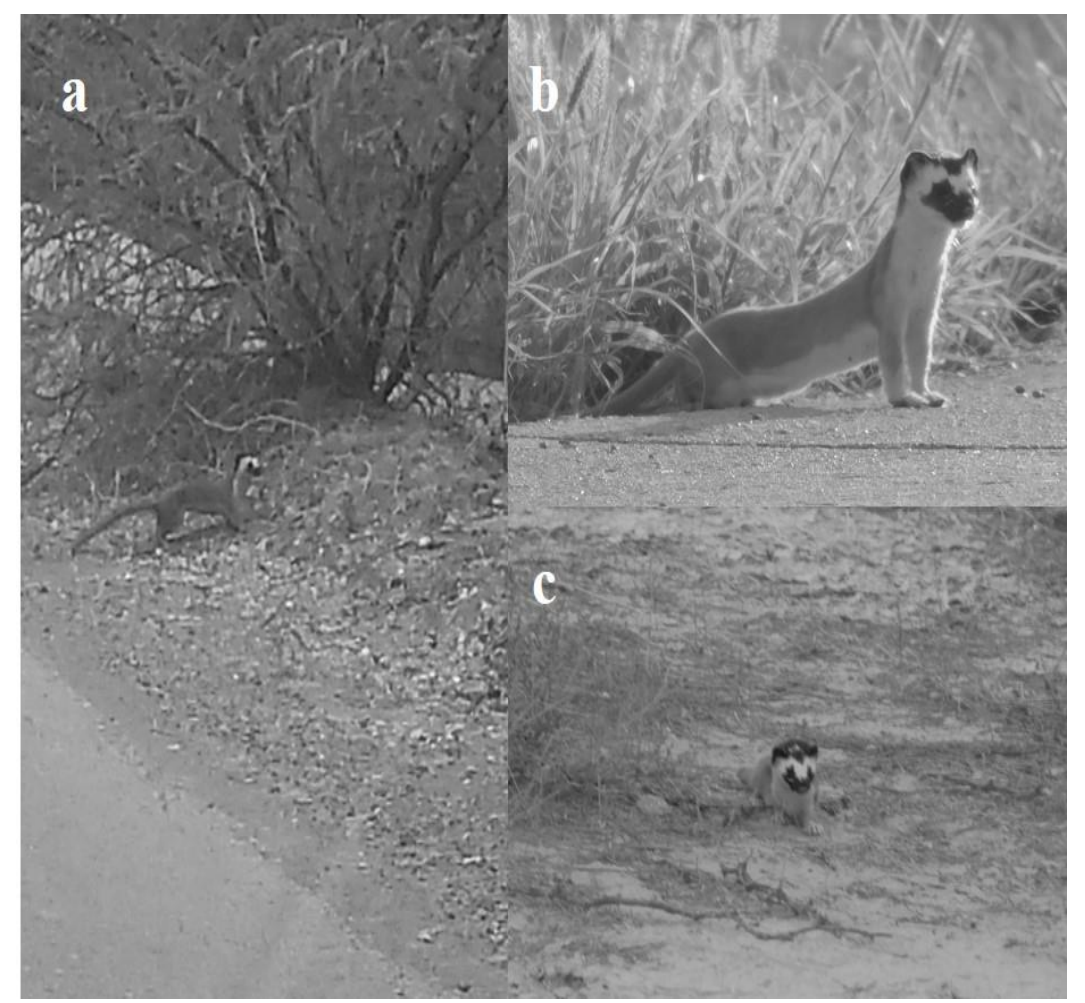

Figura 2. Registros fotográficos de la comadreja de cola larga (M. frenata) en la zona semiárida al noreste de Durango, México. a) Primer registro observado cerca de la localidad URUZA-Chapingo, b) Segundo registro cercano a la localidad Bermejillo y; c) Tercer registro en la localidad La Victoria.

\section{LITERATURA CITADA}

Aranda, S. J. M. (2012) Manual para el rastreo de mamíferos silvestres de México. Comisión Nacional para el Conocimiento y Uso de la Biodiversidad (CONABIO). México, 255 pp. https://doi.org/10.5962/bhl.title.113211

Baker, R. H., Greer, K. (1962) Mammals of the Mexican state of Durango. Publications of the MuseumMichigan State University. Biological Series, 2, 29-154.

Ceballos, G., Galindo, C. (1984) Mamíferos silvestres de la Cuenca de México. Limusa, D. F., México.

Ceballos, G., Blanco, S., González, C., Martínez, E. (2006) Mustela frenata (Comadreja). Distribución potencial. Comisión Nacional para el Conocimiento y Uso de la Biodiversidad, México. Disponible en:

http://www.conabio.gob.mx/informacion/metadata/gis/mus_frengw.xml?_httpcache=yes\&_xsl=/d b/metadata/xsl/fgdc_html.xsl\&_indent=no (consultado en enero, 2018).

CONANP (2006) Programa de Conservación y Manejo de la Reserva de la Biosfera Mapimí. Comisión Nacional de Áreas Naturales Protegidas. México, D. F., 179 pp. 
Dirzo, R., Young, H. S., Galetti, M., Ceballos, G., Isaac, N. J., Collen, B. (2014) Defaunation in the Anthropocene. Science, 345, 401-406. https://doi.org/10.1126/science.1251817

Elizalde-Arellano, C., López-Vidal, J. C., Hernández-García, L., Laundré, J. W., Cervantes-Reza, F., Morales-Mejía, F. M., Ramírez-Vargas, M., Dávila-Galaviz, L. F., González-Romero, A., Alonso-Spilsbury, M. (2014) Registro de presencia y actividades de algunos mamíferos en el Desierto Chihuahuense, México. Therya, 5, 793-816. https://doi.org/10.12933/therya-14-252

Elsasser, S. C., Parker, G. H. (2008) Morphometric criteria for distinguishing species and age-cohorts of Ermine (Mustela erminea) and long-tailed weasel (M. frenata). Acta Zoologica Academiae Scientiarum Hungaricae, 54 (1), 75-88.

Feldhamer, G. A., Drickamer, L. C., Vessey, S. H., Merritt, J. F., Krajewski, C. (2007) Mammalogy: adaptation, diversity, ecology. The Johns Hopkins University Press. Baltimore, Maryland, United States of America, $613 \mathrm{pp}$.

García, E. (2004) Modificaciones al Sistema de Clasificación Climática de Köppen. Serie Libros. Méx. 90 pp.

García-Mendoza, D. F., López-González, C. (2013) A checklist of the mammals (Mammalia) from Durango, western Mexico. Check List, 9, 313-322. http://dx.doi.org/10.15560/9.2.313

Gehring, T. M., Swihart, R. K. (2004) Home range and movements of long-tailed weasels in a landscape fragmented by agriculture. Journal of Mammalogy, 85, 79-86. https://doi.org/10.1644/1545-1542(2004)085<0079:HRAMOL>2.0

González E., M. S., González E., M., Márquez L., M. A. (2006) Vegetación y ecorregiones de Durango. CIIDIR Unidad Durango. Instituto Politécnico Nacional. Durango, Durango. México. 165 pp.

Helgen, K., Reid, F. (2016) Mustela frenata. The IUCN Red List of Threatened Species 2016: e.T41654A45213820. Available at: http://dx.doi.org/10.2305/IUCN.UK.20161.RLTS.T41654A45213820.en. (accessed on March 2018).

INEGI (2015) Instituto Nacional de Estadística Geografía e Informática. Conjunto nacional de Uso de Suelo y Vegetación, escala 1:250000, serie V. Disponible en: http://www.inegi.org.mx/geo/contenidos/recnat/usosuelo/Default.aspx (consultado el 10 de febrero, 2018).

Martínez-Hernández, A., Rodríguez-Luévano, N. V. (2017) Guía para la identificación de individuos durante la ejecución del programa de protección y conservación de fauna silvestre. Villanueva Solar y Parque Solar Villanueva Tres. CESCA. 14 pp.

SEMARNAT (2010) Norma Oficial Mexicana NOM-059-SEMARNAT-2010, Protección ambientalEspecies nativas de México de flora y fauna silvestres-Categorías de riesgo y especificaciones para su inclusión, exclusión o cambio-Lista de especies en riesgo. Diario Oficial de la Federación. México.

Servín-Martínez, J. I., Huxley, C., Chacón, E., Alonso, N., González-Trápaga, R. (1998) Los mamíferos del estado de Durango, México. Instituto de Ecología, A.C. Centro Regional-Durango. Comisión Nacional para el uso y Estudio de la Biodiversidad. México, D.F. 49 pp.

Servín-Martínez J. I., Ramos-Rivera P. (2017) Los mamíferos del estado de Durango, México. Comisión nacional para el conocimiento y uso de la biodiversidad. Disponible en: https://doi.org/10.15468/jt3nwh (consultado en enero, 2018).

Sheffield, S. R., Thomas, H. H. (1997) Mustela frenata. Mammalian Species, 570, 1-9. https://doi.org/10.1644/0.570.1.

Vaughan, T. A. (1972) Mammalogy. W. B. Saunders Company, Philadelphia, Pennsylvania, United States of America. 466 pp. 